\title{
Quantitative uniqueness for the power of the Laplacian with singular coefficients
}

\author{
Ching-Lung Lin, Sei Nagayasu And Jenn-NAn WAng
}

\begin{abstract}
In this paper we study the local behavior of a solution to the $l$-th power of the Laplacian with singular coefficients in lower order terms. We obtain a bound on the vanishing order of the nontrivial solution. Our proofs use Carleman estimates with carefully chosen weights. We will derive appropriate three-sphere inequalities and apply them to obtain doubling inequalities and the maximal vanishing order.
\end{abstract}

Mathematics Subject Classification (2010): 35J15 (primary); 35A02 (secondary).

\section{Introduction}

Assume that $\Omega$ is a connected open set containing 0 in $\mathbb{R}^{n}$ for $n \geq 2$. In this paper we are interested in the local behavior of $u$ satisfying the following differential inequality:

$$
\left|\Delta^{l} u\right| \leq K_{0} \sum_{|\alpha| \leq l-1}|x|^{-2 l+|\alpha|}\left|D^{\alpha} u\right|+K_{0} \sum_{|\alpha|=l}^{[3 l / 2]}|x|^{-2 l+|\alpha|+\epsilon}\left|D^{\alpha} u\right|,
$$

where $0<\epsilon<1 / 2$ and $[h]=k \in \mathbb{Z}$ when $k \leq h<k+1$. For (1.1), a strong unique continuation was proved by the first author [9]. A similar result for the power of the Laplacian with lower derivatives up to $l$-th order can be found in [2]. On the other hand, a unique continuation property for the $l$-th power of the Laplacian with the same order of lower derivatives as in (1.1) was given in [12]. The results mentioned above concern only the qualitative behavior of the solution. In other words, they show that if $u$ vanishes at 0 in infinite order or $u$ vanishes in an open subset of $\Omega$, then $u$ must vanish identically in $\Omega$.

The first and third authors were partially supported by the National Science Council of Taiwan. Received November 18, 2009; accepted in revised form April 22, 2010. 
The aim of this paper is to study the strong unique continuation from a quantitative viewpoint. Namely, we are interested in the maximal vanishing order at 0 of any nontrivial solution to (1.1). It is worth mentioning that quantitative estimates of the strong unique continuation are useful in studying the nodal sets of eigenfunctions [3], or solutions of second-order elliptic equations [7, 11], or the inverse problem [1].

Perhaps, for the quantitative uniqueness problem, the most popular technique, introduced by Garofalo and Lin $[4,5]$, is to use the frequency function related to the solution. This method works quite efficiently for second-order strongly elliptic operators. However, this method cannot be applied to (1.1). Another method to derive quantitative estimates of the strong unique continuation is based on Carleman estimates, which was first initiated by Donnelly and Fefferman [3] where they studied the maximal vanishing order of the eigenfunction with respect to the corresponding eigenvalue on a compact smooth Riemannian manifold. Their method does not work for (1.1) either.

Recently, the first and third authors and Nakamura [10] introduced a method based on appropriate Carleman estimates to prove a quantitative uniqueness for second-order elliptic operators with sharp singular coefficients in lower order terms. A key strategy of our method is to derive three-sphere inequalities and then apply them to obtain doubling inequalities and the maximal vanishing order. Both steps require delicate choices of cut-off functions. Nevertheless, this method is quite versatile and can be adapted to treat many equations or even systems. The present work is an application of the ideas of [10] to the $l$-th power of the Laplacian with singular coefficients. The power $l=2$ is the most interesting and useful case. It corresponds to the biharmonic operator with third-order derivatives. Our work provides a quantitative estimate of the strong unique continuation for this equation. To our best knowledge, this quantitative estimate has not been derived before.

Before stating the main results of the paper, we want to remark that if the right-hand side of (1.1) contains only $l$-th (or lower) order derivatives and has mild singular coefficients, then the Carleman estimate (3.1) alone is sufficient to derive doubling inequalities. However, if the highest order of the right-hand side of (1.1) is strictly larger than $l$, even with bounded coefficients, (3.1) is not enough to deduce doubling inequalities. The reason is that the constant in (3.1) behaves like $m^{2 l-2|\alpha|}$ for $|\alpha| \leq 2 l$ and it decays to zero when $|\alpha|>l$. The trick to overcome this difficulty is to use three-sphere inequalities, which is another form of a quantitative uniqueness estimate.

We now state the main results of the paper. Assume that $B_{R_{0}^{\prime}} \subset \Omega$ for some $R_{0}^{\prime}>0$.

Theorem 1.1. There exists a positive number $\tilde{R}_{0}<e^{-1 / 2}$ such that if $0<r_{1}<$ $r_{2}<r_{3} \leq R_{0}^{\prime}<1$ and $r_{1} / r_{3}<r_{2} / r_{3}<\tilde{R}_{0}$, then

$$
\int_{|x|<r_{2}}|u|^{2} d x \leq C\left(\int_{|x|<r_{1}}|u|^{2} d x\right)^{\tau}\left(\int_{|x|<r_{3}}|u|^{2} d x\right)^{1-\tau}
$$


for $u \in H^{2 l}\left(B_{R_{0}^{\prime}}\right)$ satisfying (1.1) in $B_{R_{0}^{\prime}}$, where $C$ and $0<\tau<1$ depend on $r_{1} / r_{3}$, $r_{2} / r_{3}, n, l$, and $K_{0}$.

Remark 1.2. From the proof, the constants $C$ and $\tau$ can be explicitly written as $C=\max \left\{C_{0}\left(r_{2} / r_{1}\right)^{n}, \exp \left(B \beta_{0}\right)\right\}$ and $\tau=B /(A+B)$, where $C_{0}>1$ and $\beta_{0}$ are constants depending on $n, l, K_{0}$ and

$$
\begin{aligned}
& A=A\left(r_{1} / r_{3}, r_{2} / r_{3}\right)=\left(\log \left(r_{1} / r_{3}\right)-1\right)^{2}-\left(\log \left(r_{2} / r_{3}\right)\right)^{2}, \\
& B=B\left(r_{2} / r_{3}\right)=-1-2 \log \left(r_{2} / r_{3}\right) .
\end{aligned}
$$

The explicit forms of these constants are important in the proof of Theorem 1.3.

Theorem 1.3. Let $u \in H_{\mathrm{loc}}^{2 l}(\Omega)$ be a nonzero solution to (1.1). Then we can find a constant $R_{2}$ (depending on $n, l, \epsilon, K_{0}$ ) and a constant $m_{1}$ (depending on $\left.n, l, \epsilon, K_{0},\|u\|_{L^{2}\left(|x|<R_{2}^{2}\right)} /\|u\|_{L^{2}\left(|x|<R_{2}^{4}\right)}\right)$ such that if $0<r \leq R_{3}$, then

$$
C_{3} r^{m_{1}} \leq \int_{|x|<r}|u|^{2} d x
$$

where $R_{3}$ is a positive constants depending on $n, l, \epsilon, K_{0}, m_{1}$ and $C_{3}$ is a positive constants depending on $n, l, \epsilon, K_{0}, m_{1}, u$.

Theorem 1.4. Let $u \in H_{\mathrm{loc}}^{2 l}(\Omega)$ be a nonzero solution to (1.1). Then there exist positive constants $R_{4}$ (depending on $n, l, \epsilon, K_{0}, m_{1}$ ) and $C_{4}$ (depending on $\left.n, l, \epsilon, K_{0}, m_{1}\right)$ such that if $0<r \leq R_{4}$, then

$$
\int_{|x| \leq 2 r}|u|^{2} d x \leq C_{4} \int_{|x| \leq r}|u|^{2} d x
$$

where $m_{1}$ is the constant obtained in Theorem 1.3.

The rest of the paper is devoted to the proofs of Theorems 1.1-1.4.

\section{Three-sphere inequalities}

In this section we will prove Theorem 1.1. To begin, we recall a Carleman estimate with weight $\varphi_{\beta}=\varphi_{\beta}(x)=\exp \left(\frac{\beta}{2}(\log |x|)^{2}\right)$ given in [9]. 
Lemma 2.1 ([9, Corollary 3.3]). There exist a sufficiently large number $\beta_{0}>0$ and a sufficiently small number $r_{0}>0$, depending on $n$ and $l$, such that for all $u \in U_{r_{0}}$ with $0<r_{0}<e^{-1}, \beta \geq \beta_{0}$, we have that

$$
\begin{aligned}
& \sum_{|\alpha| \leq 2 l} \beta^{3 l-2|\alpha|} \int \varphi_{\beta}^{2}|x|^{2|\alpha|-n}(\log |x|)^{2 l-2|\alpha|}\left|D^{\alpha} u\right|^{2} d x \\
& \leq \tilde{C}_{0} \int \varphi_{\beta}^{2}|x|^{4 l-n}\left|\Delta^{l} u\right|^{2} d x,
\end{aligned}
$$

where $U_{r_{0}}=\left\{u \in C_{0}^{\infty}\left(\mathbb{R}^{n} \backslash\{0\}\right): \operatorname{supp}(u) \subset B_{r_{0}}\right\}$ and $\tilde{C}_{0}$ is a positive constant depending on $n$ and $l$. Here $e=\exp (1)$.

Remark 2.2. The estimate (2.1) in Lemma 2.1 remains valid if we assume $u \in$ $H_{\text {loc }}^{2 l}\left(\mathbb{R}^{n} \backslash\{0\}\right)$ with compact support. This can be easily obtained by cutting off $u$ for small $|x|$ and regularizing.

Proof. We first consider the case where $0<r_{1}<r_{2}<R<1 / e$ and $B_{R} \subset \Omega$. The constant $R$ will be chosen later. To use the estimate (2.1), we need to cut off $u$. So let $\xi(x) \in C_{0}^{\infty}\left(\mathbb{R}^{n}\right)$ satisfy $0 \leq \xi(x) \leq 1$ and

$$
\xi(x)= \begin{cases}0, & |x| \leq r_{1} / e \\ 1, & r_{1} / 2<|x|<e r_{2} \\ 0, & |x| \geq 3 r_{2}\end{cases}
$$

It is easy to check that for all multiindex $\alpha$

$$
\left\{\begin{array}{l}
\left|D^{\alpha} \xi\right|=O\left(r_{1}^{-|\alpha|}\right) \text { for all } r_{1} / e \leq|x| \leq r_{1} / 2 \\
\left|D^{\alpha} \xi\right|=O\left(r_{2}^{-|\alpha|}\right) \text { for all } e r_{2} \leq|x| \leq 3 r_{2}
\end{array}\right.
$$

On the other hand, repeating [8, proof of Corollary 17.1.4], we can show that

$$
\left.\left.\int_{a_{1} r<|x|<a_{2} r}|| x\right|^{|\alpha|} D^{\alpha} u\right|^{2} d x \leq C^{\prime} \int_{a_{3} r<|x|<a_{4} r}|u|^{2} d x, \quad|\alpha| \leq 2 l,
$$

for all $0<a_{3}<a_{1}<a_{2}<a_{4}$ such that $B_{a_{4} r} \subset \Omega$, where the constant $C^{\prime}$ is independent of $r$ and $u$. 
Notice that the commutator $\left[\Delta^{l}, \xi\right]$ is a $2 l-1$ order differential operator. Applying (2.1) to $\xi u$ and using (1.1), (2.2), (2.3) implies

$$
\begin{aligned}
& \sum_{|\alpha| \leq 2 l} \beta^{3 l-2|\alpha|} \int_{r_{1} / 2<|x|<e r_{2}} \varphi_{\beta}^{2}|x|^{2|\alpha|-n}(\log |x|)^{2 l-2|\alpha|}\left|D^{\alpha} u\right|^{2} d x \\
& \leq \sum_{|\alpha| \leq 2 l} \beta^{3 l-2|\alpha|} \int \varphi_{\beta}^{2}|x|^{2|\alpha|-n}(\log |x|)^{2 l-2|\alpha|}\left|D^{\alpha}(\xi u)\right|^{2} d x \\
& \leq \tilde{C}_{0} \int \varphi_{\beta}^{2}|x|^{4 l-n}\left|\Delta^{l}(\xi u)\right|^{2} d x \\
& \leq 2 \tilde{C}_{0} \int \varphi_{\beta}^{2}|x|^{4 l-n} \xi^{2}\left(K_{0} \sum_{|\alpha| \leq l-1}|x|^{-2 l+|\alpha|}\left|D^{\alpha} u\right|+K_{0} \sum_{|\alpha|=l}^{[3 l / 2]}|x|^{-2 l+|\alpha|+\epsilon}\left|D^{\alpha} u\right|\right)^{2} d x \\
& +2 \tilde{C}_{0} \int \varphi_{\beta}^{2}|x|^{4 l-n}\left|\left[\Delta^{l}, \xi\right] u\right|^{2} d x \\
& \leq \tilde{C}_{1}\left\{\int_{r_{1} / 2<|x|<e r_{2}} \varphi_{\beta}^{2}\left(\sum_{|\alpha| \leq l-1}|x|^{2|\alpha|-n}\left|D^{\alpha} u\right|^{2}+\sum_{|\alpha|=l}^{[3 l / 2]}|x|^{2|\alpha|-n+2 \epsilon}\left|D^{\alpha} u\right|^{2}\right) d x\right. \\
& +\int_{r_{1} / e<|x|<r_{1} / 2} \varphi_{\beta}^{2} \sum_{|\alpha| \leq 2 l-1}|x|^{2|\alpha|-n}\left|D^{\alpha} u\right|^{2} d x \\
& \left.+\int_{e r_{2}<|x|<3 r_{2}} \varphi_{\beta}^{2} \sum_{|\alpha| \leq 2 l-1}|x|^{2|\alpha|-n}\left|D^{\alpha} u\right|^{2} d x\right\} \\
& \leq \tilde{C}_{2}\left\{\int_{r_{1} / 2<|x|<e r_{2}} \varphi_{\beta}^{2}\left(\sum_{|\alpha| \leq l-1}|x|^{2|\alpha|-n}\left|D^{\alpha} u\right|^{2}+\sum_{|\alpha|=l}^{[3 l / 2]}|x|^{2|\alpha|-n+2 \epsilon}\left|D^{\alpha} u\right|^{2}\right) d x\right. \\
& +\left.\left.r_{1}^{-n} \varphi_{\beta}^{2}\left(r_{1} / e\right) \int_{r_{1} / e<|x|<r_{1} / 2} \sum_{|\alpha| \leq 2 l-1}|| x\right|^{|\alpha|} D^{\alpha} u\right|^{2} d x \\
& \left.+\left.\left.r_{2}^{-n} \varphi_{\beta}^{2}\left(e r_{2}\right) \int_{e r_{2}<|x|<3 r_{2}} \sum_{|\alpha| \leq 2 l-1}|| x\right|^{|\alpha|} D^{\alpha} u\right|^{2} d x\right\} \\
& \leq \tilde{C}_{3}\left\{\int_{r_{1} / 2<|x|<e r_{2}} \varphi_{\beta}^{2}\left(\sum_{|\alpha| \leq l-1}|x|^{2|\alpha|-n}\left|D^{\alpha} u\right|^{2}+\sum_{|\alpha|=l}^{[3 l / 2]}|x|^{2|\alpha|-n+2 \epsilon}\left|D^{\alpha} u\right|^{2}\right) d x\right. \\
& \left.+r_{1}^{-n} \varphi_{\beta}^{2}\left(r_{1} / e\right) \int_{r_{1} / 4<|x|<r_{1}}|u|^{2} d x+r_{2}^{-n} \varphi_{\beta}^{2}\left(e r_{2}\right) \int_{2 r_{2}<|x|<4 r_{2}}|u|^{2} d x\right\},
\end{aligned}
$$

where $\tilde{C}_{1}, \tilde{C}_{2}$, and $\tilde{C}_{3}$ are independent of $r_{1}, r_{2}$, and $u$. 
We now choose $r_{0}<e^{-\epsilon^{-1}([3 l / 2]-l)-1}$ small enough that

$$
\left\{\begin{array}{l}
\left(\log \left(e r_{0}\right)\right)^{-2} \leq \frac{1}{2 \tilde{C}_{3}} \\
\left(e r_{0}\right)^{2 \epsilon}\left(\log \left(e r_{0}\right)\right)^{2([3 l / 2]-l)} \leq \frac{1}{2 \tilde{C}_{3}} .
\end{array}\right.
$$

Letting $R \leq r_{0}$ and $\beta \geq \beta_{0} \geq \max \left\{2 \tilde{C}_{3}, 1\right\}$, we can absorb the integral over $r_{1} / 2<|x|<e r_{2}$ on the right-hand side of (2.4) into its left-hand side to obtain

$$
\begin{aligned}
& \int_{r_{1} / 2<|x|<e r_{2}} \varphi_{\beta}^{2}\left(\sum_{|\alpha| \leq l-1}|x|^{2|\alpha|-n}\left|D^{\alpha} u\right|^{2}+\sum_{|\alpha|=l}^{[3 l / 2]}|x|^{2|\alpha|-n+2 \epsilon}\left|D^{\alpha} u\right|^{2}\right) d x \\
& \leq \tilde{C}_{4}\left\{r_{1}^{-n} \varphi_{\beta}^{2}\left(r_{1} / e\right) \int_{r_{1} / 4<|x|<r_{1}}|u|^{2} d x+r_{2}^{-n} \varphi_{\beta}^{2}\left(e r_{2}\right) \int_{2 r_{2}<|x|<4 r_{2}}|u|^{2} d x\right\},
\end{aligned}
$$

where $\tilde{C}_{4}=1 / \tilde{C}_{3}$. Using (2.5) we have that

$$
\begin{aligned}
& r_{2}^{-n} \varphi_{\beta}^{2}\left(r_{2}\right) \int_{r_{1} / 2<|x|<r_{2}}|u|^{2} d x \\
& \leq \int_{r_{1} / 2<|x|<e r_{2}} \varphi_{\beta}^{2}|x|^{-n}|u|^{2} d x \\
& \leq \tilde{C}_{4}\left\{r_{1}^{-n} \varphi_{\beta}^{2}\left(r_{1} / e\right) \int_{r_{1} / 4<|x|<r_{1}}|u|^{2} d x+r_{2}^{-n} \varphi_{\beta}^{2}\left(e r_{2}\right) \int_{2 r_{2}<|x|<4 r_{2}}|u|^{2} d x\right\} .
\end{aligned}
$$

Dividing $r_{2}^{-n} \varphi_{\beta}^{2}\left(r_{2}\right)$ both sides of (2.6) we get

$$
\begin{aligned}
& \int_{r_{1} / 2<|x|<r_{2}}|u|^{2} d x \\
& \leq \tilde{C}_{4}\left\{\left(r_{2} / r_{1}\right)^{n}\left[\varphi_{\beta}^{2}\left(r_{1} / e\right) / \varphi_{\beta}^{2}\left(r_{2}\right)\right] \int_{r_{1} / 4<|x|<r_{1}}|u|^{2} d x\right. \\
& \left.\quad+\left[\varphi_{\beta}^{2}\left(e r_{2}\right) / \varphi_{\beta}^{2}\left(r_{2}\right)\right] \int_{2 r_{2}<|x|<4 r_{2}}|u|^{2} d x\right\} \\
& \leq \tilde{C}_{5}\left\{\left(r_{2} / r_{1}\right)^{n}\left[\varphi_{\beta}^{2}\left(r_{1} / e\right) / \varphi_{\beta}^{2}\left(r_{2}\right)\right] \int_{|x|<r_{1}}|u|^{2} d x\right. \\
& \left.\quad+\left(r_{2} / r_{1}\right)^{n}\left[\varphi_{\beta}^{2}\left(e r_{2}\right) / \varphi_{\beta}^{2}\left(r_{2}\right)\right] \int_{|x|<4 r_{2}}|u|^{2} d x\right\},
\end{aligned}
$$

where $\tilde{C}_{5}=\max \left\{\tilde{C}_{4}, 1\right\}$. With such choice of $\tilde{C}_{5}$, we can see that

$$
\tilde{C}_{5}\left(r_{2} / r_{1}\right)^{n}\left[\varphi_{\beta}^{2}\left(r_{1} / e\right) / \varphi_{\beta}^{2}\left(r_{2}\right)\right]>1
$$


for all $0<r_{1}<r_{2}$. Adding $\int_{|x|<r_{1} / 2}|u|^{2} d x$ to both sides of (2.7) and choosing $r_{2} \leq R=\min \left\{r_{0}, 1 / 4\right\}$, we get

$$
\begin{aligned}
\int_{|x|<r_{2}}|u|^{2} d x \leq & 2 \tilde{C}_{5}\left(r_{2} / r_{1}\right)^{n}\left[\varphi_{\beta}^{2}\left(r_{1} / e\right) / \varphi_{\beta}^{2}\left(r_{2}\right)\right] \int_{|x|<r_{1}}|u|^{2} d x \\
& +2 \tilde{C}_{5}\left(r_{2} / r_{1}\right)^{n}\left[\varphi_{\beta}^{2}\left(e r_{2}\right) / \varphi_{\beta}^{2}\left(r_{2}\right)\right] \int_{|x|<1}|u|^{2} d x .
\end{aligned}
$$

Setting

$$
\begin{aligned}
& A=\beta^{-1} \log \left[\varphi_{\beta}^{2}\left(r_{1} / e\right) / \varphi_{\beta}^{2}\left(r_{2}\right)\right]=\left(\log r_{1}-1\right)^{2}-\left(\log r_{2}\right)^{2}>0, \\
& B=-\beta^{-1} \log \left[\varphi_{\beta}^{2}\left(e r_{2}\right) / \varphi_{\beta}^{2}\left(r_{2}\right)\right]=-1-2 \log r_{2}>0,
\end{aligned}
$$

inequality (2.8) becomes

$$
\begin{aligned}
& \int_{|x|<r_{2}}|u|^{2} d x \\
& \leq 2 \tilde{C}_{5}\left(r_{2} / r_{1}\right)^{n}\left\{\exp (A \beta) \int_{|x|<r_{1}}|u|^{2} d x+\exp (-B \beta) \int_{|x|<1}|u|^{2} d x\right\} .
\end{aligned}
$$

To further simplify the terms on the right-hand side of (2.9), we consider two cases. If $\int_{|x|<r_{1}}|u|^{2} d x \neq 0$ and

$$
\exp \left(A \beta_{0}\right) \int_{|x|<r_{1}}|u|^{2} d x<\exp \left(-B \beta_{0}\right) \int_{|x|<1}|u|^{2} d x,
$$

then we can pick $\beta>\beta_{0}$ such that

$$
\exp (A \beta) \int_{|x|<r_{1}}|u|^{2} d x=\exp (-B \beta) \int_{|x|<1}|u|^{2} d x .
$$

Using such a $\beta$, we obtain from (2.9) that

$$
\begin{aligned}
\int_{|x|<r_{2}}|u|^{2} d x & \leq 4 \tilde{C}_{5}\left(r_{2} / r_{1}\right)^{n} \exp (A \beta) \int_{|x|<r_{1}}|u|^{2} d x \\
& =4 \tilde{C}_{5}\left(r_{2} / r_{1}\right)^{n}\left(\int_{|x|<r_{1}}|u|^{2} d x\right)^{\frac{B}{A+B}}\left(\int_{|x|<1}|u|^{2} d x\right)^{\frac{A}{A+B}} .
\end{aligned}
$$

If $\int_{|x|<r_{1}}|u|^{2} d x=0$, then it follows from (2.9) that

$$
\int_{|x|<r_{2}}|u|^{2} d x=0
$$


since we can take $\beta$ arbitrarily large. The three-sphere inequality obviously holds. On the other hand, if

$$
\exp \left(-B \beta_{0}\right) \int_{|x|<1}|u|^{2} d x \leq \exp \left(A \beta_{0}\right) \int_{|x|<r_{1}}|u|^{2} d x,
$$

then we have

$$
\begin{aligned}
\int_{|x|<r_{2}}|u|^{2} d x & \leq\left(\int_{|x|<1}|u|^{2} d x\right)^{\frac{B}{A+B}}\left(\int_{|x|<1}|u|^{2} d x\right)^{\frac{A}{A+B}} \\
& \leq \exp \left(B \beta_{0}\right)\left(\int_{|x|<r_{1}}|u|^{2} d x\right)^{\frac{B}{A+B}}\left(\int_{|x|<1}|u|^{2} d x\right)^{\frac{A}{A+B}} .
\end{aligned}
$$

Putting together (2.10), (2.11), and setting $\tilde{C}_{6}=\max \left\{4 \tilde{C}_{5}\left(r_{2} / r_{1}\right)^{n}, \exp \left(B \beta_{0}\right)\right\}$, we arrive at

$$
\int_{|x|<r_{2}}|u|^{2} d x \leq \tilde{C}_{6}\left(\int_{|x|<r_{1}}|u|^{2} d x\right)^{\frac{B}{A+B}}\left(\int_{|x|<1}|u|^{2} d x\right)^{\frac{A}{A+B}} .
$$

Now for the general case, we take $\tilde{R}_{0}=R$ and consider $0<r_{1}<r_{2}<r_{3}$ with $r_{1} / r_{3}<r_{2} / r_{3} \leq \tilde{R}_{0}$. By scaling, i.e., defining $\widehat{u}(y):=u\left(r_{3} y\right)$, we derive from (2.12) that

$$
\int_{|y|<r_{2} / r_{3}}|\widehat{u}|^{2} d y \leq C\left(\int_{|y|<r_{1} / r_{3}}|\widehat{u}|^{2} d y\right)^{\tau}\left(\int_{|y|<1}|\widehat{u}|^{2} d y\right)^{1-\tau},
$$

where $\tau=B /(A+B)$ with

$$
\begin{aligned}
& A=A\left(r_{1} / r_{3}, r_{2} / r_{3}\right)=\left(\log \left(r_{1} / r_{3}\right)-1\right)^{2}-\left(\log \left(r_{2} / r_{3}\right)\right)^{2}, \\
& B=B\left(r_{2} / r_{3}\right)=-1-2 \log \left(r_{2} / r_{3}\right),
\end{aligned}
$$

and $C=\max \left\{4 \tilde{C}_{5}\left(r_{2} / r_{1}\right)^{n}, \exp \left(B \beta_{0}\right)\right\}$. Note that $\tilde{C}_{5}$ can be chosen independent of the scaling factor $r_{3}$ provided $r_{3}<1$. Replacing the variable $y=x / r_{3}$ in (2.13) gives

$$
\int_{|x|<r_{2}}|u|^{2} d x \leq C\left(\int_{|x|<r_{1}}|u|^{2} d x\right)^{\tau}\left(\int_{|x|<r_{3}}|u|^{2} d x\right)^{1-\tau} .
$$

This concludes the proof.

\section{Doubling inequalities and maximal vanishing order}

In this section we prove Theorem 1.3 and Theorem 1.4. We begin with another Carleman estimate derived in [9, Lemma 2.1]: for any $u \in C_{0}^{\infty}\left(\mathbb{R}^{n} \backslash\{0\}\right)$ and for any $m \in\{k+1 / 2, k \in \mathbb{N}\}$, we have the following estimate

$$
\sum_{|\alpha| \leq 2 l} \int m^{2 l-2|\alpha|}|x|^{-2 m+2|\alpha|-n}\left|D^{\alpha} u\right|^{2} d x \leq C \int|x|^{-2 m+4 l-n}\left|\triangle^{l} u\right|^{2} d x,
$$

where $C$ depends only on the dimension $n$ and the power $l$. 
Remark 3.1. Using the cut-off function and regularization, the estimate (3.1) remains valid for any fixed $m$ if $u \in H_{\text {loc }}^{2 l}\left(\mathbb{R}^{n} \backslash\{0\}\right)$ with compact support.

Proof. In view of Remark 3.1, we can apply (3.1) to the function $\chi u$ with $\chi(x) \in$ $C_{0}^{\infty}\left(\mathbb{R}^{n} \backslash\{0\}\right)$. Thus, we define $\chi(x) \in C_{0}^{\infty}\left(\mathbb{R}^{n} \backslash\{0\}\right)$ as

$$
\chi(x)=\left\{\begin{array}{lll}
0 & \text { if } & |x| \leq \delta / 3 \\
1 & \text { if } & \delta / 2 \leq|x| \leq\left(R_{0}+1\right) R_{0} R / 4=r_{4} R \\
0 & \text { if } & 2 r_{4} R \leq|x|
\end{array}\right.
$$

where $\delta \leq R_{0}^{2} R / 4, R_{0}>0$ is a small number which will be chosen later and $R<1$ is sufficiently small. Here the number $R$ is not yet fixed and is given by $R=(\gamma m)^{-l / 2 \epsilon}$, where $\gamma>0$ is a large constant which will be determined later. Using the estimate (3.1) and equation (1.1), we can derive that

$$
\begin{aligned}
& \sum_{|\alpha| \leq 2 l} \int_{\delta / 2 \leq|x| \leq r_{4} R} m^{2 l-2|\alpha|}|x|^{-2 m+2|\alpha|-n}\left|D^{\alpha} u\right|^{2} d x \\
\leq & \sum_{|\alpha| \leq 2 l} \int m^{2 l-2|\alpha|}|x|^{-2 m+2|\alpha|-n}\left|D^{\alpha}(\chi u)\right|^{2} d x \\
\leq & C \int_{|x|^{-2 m+4 l-n}\left|\Delta^{l}(\chi u)\right|^{2} d x} \\
= & C \int_{\delta / 2 \leq|x| \leq r_{4} R}|x|^{-2 m+4 l-n}\left|\Delta^{l} u\right|^{2} d x+C \int_{|x|>r_{4} R}|x|^{-2 m+4 l-n}\left|\Delta^{l}(\chi u)\right|^{2} d x \\
& +C \int_{\delta / 3 \leq|x| \leq \delta / 2}|x|^{-2 m+4 l-n}\left|\Delta^{l}(\chi u)\right|^{2} d x \\
\leq & C^{\prime} K_{0}^{2} \int_{\delta / 2 \leq|x| \leq r_{4} R}\left(\sum_{|\alpha| \leq l-1}|x|^{2|\alpha|-n-2 m}\left|D^{\alpha} u\right|^{2}+\sum_{|\alpha|=l}^{[3 l / 2]}|x|^{2|\alpha|-n-2 m+2 \epsilon}\left|D^{\alpha} u\right|^{2}\right) d x \\
& +C \int_{|x|>r_{4} R}|x|^{-2 m+4 l-n}\left|\Delta^{l}(\chi u)\right|^{2} d x+C \int_{\delta / 3 \leq|x| \leq \delta / 2}^{|x|^{-2 m+4 l-n}\left|\Delta^{l}(\chi u)\right|^{2} d x} \\
\leq & C^{\prime} K_{0}^{2}\left(r_{4} R\right)^{2 \epsilon} \int_{\delta / 2 \leq|x| \leq r_{4} R} \sum_{|\alpha|=l}|x|^{2|\alpha|-n-2 m}\left|D^{\alpha} u\right|^{2} d x \\
& +C^{\prime} K_{0}^{2} \int_{\delta / 2 \leq|x| \leq r_{4} R} \sum_{|\alpha| \leq l-1}|x|^{2|\alpha|-n-2 m}\left|D^{\alpha} u\right|^{2} d x \\
& +C \int_{|x|>r_{4} R}|x|^{-2 m+4 l-n}\left|\Delta^{l}(\chi u)\right|^{2} d x+C \int_{\delta / 3 \leq|x| \leq \delta / 2}|x|^{-2 m+4 l-n}\left|\Delta^{l}(\chi u)\right|^{2} d x,
\end{aligned}
$$

where the constant $C^{\prime}$ depends on $n$ and $l$. 
By carefully checking the terms on both sides of (3.2), we now choose $\gamma \geq$ $\left(2 C^{\prime} K_{0}^{2}\right)^{1 / l}$ and thus

$$
R^{2 \epsilon}=(\gamma m)^{-l} \leq \frac{m^{-l}}{2 C^{\prime} K_{0}^{2}}
$$

Hence, choosing $R_{0}<1$ (which suffices to guarantee that $r_{4}^{2 / \epsilon}=R_{0}^{2 \epsilon}\left(R_{0}+\right.$ $\left.1)^{2 \epsilon} / 4^{2 \epsilon}<1\right)$ and $m$ such that $m^{2}>2 C^{\prime} K_{0}^{2}$, we can remove the first two terms on the right-hand side of the last inequality in (3.2) and obtain

$$
\begin{aligned}
& \sum_{|\alpha| \leq 2 l} \int_{\delta / 2 \leq|x| \leq r_{4} R} m^{2 l-2|\alpha|}|x|^{-2 m+2|\alpha|-n}\left|D^{\alpha} u\right|^{2} d x \\
& \leq 2 C \int_{\delta / 3<|x|<\delta / 2}|x|^{-2 m+4 l-n}\left|\Delta^{l}(\chi u)\right|^{2} d x \\
& \quad+2 C \int_{r_{4} R<|x|<2 r_{4} R}|x|^{-2 m+4 l-n}\left|\Delta^{l}(\chi u)\right|^{2} d x .
\end{aligned}
$$

In view of the definition of $\chi$, it is easy to see that for all multiindex $\alpha$

$$
\left\{\begin{array}{l}
\left|D^{\alpha} \chi\right|=O\left(\delta^{-|\alpha|}\right) \text { for all } \delta / 3<|x|<\delta / 2 \\
\left|D^{\alpha} \chi\right|=O\left(\left(r_{4} R\right)^{-|\alpha|}\right) \text { for all } r_{4} R<|x|<2 r_{4} R
\end{array}\right.
$$

Note that $R_{0}^{2} \leq r_{4}$ provided $R_{0} \leq 1 / 3$. Therefore, using (3.4) and (2.3) in (3.3), we derive

$$
\begin{aligned}
& m^{2}(2 \delta)^{-2 m-n} \int_{\delta / 2<|x| \leq 2 \delta}|u|^{2} d x+m^{2}\left(R_{0}^{2} R\right)^{-2 m-n} \int_{2 \delta<|x| \leq R_{0}^{2} R}|u|^{2} d x \\
& \leq \sum_{|\alpha| \leq 2 l} \int_{\delta / 2 \leq|x| \leq r_{4} R} m^{2 l-2|\alpha|}|x|^{-2 m+2|\alpha|-n}\left|D^{\alpha} u\right|^{2} d x \\
& \leq C^{\prime \prime} \sum_{|\alpha| \leq 2 l} \delta^{-4 l+2|\alpha|} \int_{\delta / 3<|x|<\delta / 2}|x|^{-2 m+4 l-n}\left|D^{\alpha} u\right|^{2} d x \\
& \quad+C^{\prime \prime} \sum_{|\alpha| \leq 2 l}\left(r_{4} R\right)^{-4 l+2|\alpha|} \int_{r_{4} R<|x|<2 r_{4} R}|x|^{-2 m+4 l-n}\left|D^{\alpha} u\right|^{2} d x \\
& \leq \tilde{C}^{\prime} \delta^{-2 m-n} \int_{|x| \leq \delta}|u|^{2} d x+C^{\prime \prime}\left(r_{4} R\right)^{-2 m-n} \int_{|x| \leq R_{0} R}|u|^{2} d x,
\end{aligned}
$$

where $\tilde{C}^{\prime}=C^{\prime \prime} 3^{2 m+n}$ and $C^{\prime \prime}$ is independent of $R_{0}, R$, and $m$. 
We then add $m^{2}(2 \delta)^{-2 m-n} \int_{|x| \leq \delta / 2}|u|^{2} d x$ to both sides of (3.5) and obtain

$$
\begin{aligned}
& \frac{1}{2} m^{2}(2 \delta)^{-2 m-n} \int_{|x| \leq 2 \delta}|u|^{2} d x+m^{2}\left(R_{0}^{2} R\right)^{-2 m-n} \int_{|x| \leq R_{0}^{2} R}|u|^{2} d x \\
& =\frac{1}{2} m^{2}(2 \delta)^{-2 m-n} \int_{|x| \leq 2 \delta}|u|^{2} d x+m^{2}\left(R_{0}^{2} R\right)^{-2 m-n} \int_{|x| \leq 2 \delta}|u|^{2} d x \\
& \quad+m^{2}\left(R_{0}^{2} R\right)^{-2 m-n} \int_{2 \delta<|x| \leq R_{0}^{2} R}|u|^{2} d x \\
& \leq \frac{1}{2} m^{2}(2 \delta)^{-2 m-n} \int_{|x| \leq 2 \delta}|u|^{2} d x+\frac{1}{2} m^{2}(2 \delta)^{-2 m-n} \int_{|x| \leq 2 \delta}|u|^{2} d x \\
& \quad+m^{2}\left(R_{0}^{2} R\right)^{-2 m-n} \int_{2 \delta<|x| \leq R_{0}^{2} R}|u|^{2} d x \\
& \leq \tilde{C}^{\prime \prime} \delta^{-2 m-n} \int_{|x| \leq \delta}|u|^{2} d x+C^{\prime \prime}\left(r_{4} R\right)^{-2 m-n} \int_{|x| \leq R_{0} R}|u|^{2} d x \\
& =\tilde{C}^{\prime \prime} \delta^{-2 m-n} \int_{|x| \leq \delta}|u|^{2} d x \\
& \quad+m^{2}\left(R_{0}^{2} R\right)^{-2 m-n} C^{\prime \prime} m^{-2}\left(\frac{R_{0}^{2}}{r_{4}}\right)^{2 m+n} \int_{|x| \leq R_{0} R}|u|^{2} d x
\end{aligned}
$$

with $\tilde{C}^{\prime \prime}=\tilde{C}^{\prime}+2^{2 m+n} m^{2}$. We first observe that

$$
\begin{aligned}
C^{\prime \prime} m^{-2}\left(\frac{R_{0}^{2}}{r_{4}}\right)^{2 m+n} & =C^{\prime \prime} m^{-2}\left(\frac{4 R_{0}}{R_{0}+1}\right)^{2 m+n} \\
& \leq C^{\prime \prime} m^{-2}\left(4 R_{0}\right)^{2 m+n} \leq \exp (-2 m)
\end{aligned}
$$

for all $R_{0} \leq 1 / 16$ and $m^{2} \geq C^{\prime \prime}$. Thus, we obtain

$$
\begin{aligned}
& \frac{1}{2} m^{2}(2 \delta)^{-2 m-n} \int_{|x| \leq 2 \delta}|u|^{2} d x+m^{2}\left(R_{0}^{2} R\right)^{-2 m-n} \int_{|x| \leq R_{0}^{2} R}|u|^{2} d x \\
& \leq \tilde{C}^{\prime \prime} \delta^{-2 m-n} \int_{|x| \leq \delta}|u|^{2} d x \\
& \quad+m^{2}\left(R_{0}^{2} R\right)^{-2 m-n} \exp (-2 m) \int_{|x| \leq R_{0} R}|u|^{2} d x .
\end{aligned}
$$

It should be noted that (3.7) is valid for all $m=j+\frac{1}{2}$ with $j \in \mathbb{N}$ and $j \geq j_{0}$, where $j_{0}$ depends on $n, l, \epsilon$, and $K_{0}$. Setting $R_{j}=\left(\gamma\left(j+\frac{1}{2}\right)\right)^{-l / 2 \epsilon}$ and using the 
relation $m=(\gamma)^{-1}(R)^{-2 \epsilon / l}$, we get from (3.7) that

$$
\begin{aligned}
& \frac{1}{2} m^{2}(2 \delta)^{-2 m-n} \int_{|x| \leq 2 \delta}|u|^{2} d x+m^{2}\left(R_{0}^{2} R_{j}\right)^{-2 m-n} \int_{|x| \leq R_{0}^{2} R_{j}}|u|^{2} d x \\
& \leq \tilde{C}^{\prime \prime} \delta^{-2 m-n} \int_{|x| \leq \delta}|u|^{2} d x \\
& \quad+m^{2}\left(R_{0}^{2} R_{j}\right)^{-2 m-n} \exp \left(-2 c R_{j}^{-2 \epsilon / l}\right) \int_{|x| \leq R_{0} R_{j}}|u|^{2} d x
\end{aligned}
$$

for all $j \geq j_{0}$ and $c=\gamma^{-1}$. We now let $j_{0}$ be large enough that

$$
R_{j+1}<R_{j}<2 R_{j+1} \text { for all } j \geq j_{0} .
$$

Thus, if $R_{j+1}<R \leq R_{j}$ for $j \geq j_{0}$, we can conclude that

$$
\left\{\begin{array}{l}
\int_{|x| \leq R_{0}^{2} R}|u|^{2} d x \leq \int_{|x| \leq R_{0}^{2} R_{j}}|u|^{2} d x, \\
\exp \left(-2 c R_{j}^{-2 \epsilon / l}\right) \int_{|x| \leq R_{0} R_{j}}|u|^{2} d x \leq \exp \left(-c R^{-2 \epsilon / l}\right) \int_{|x| \leq R}|u|^{2} d x,
\end{array}\right.
$$

where we have used the inequality $R_{0} R_{j} \leq R_{j} / 16<R_{j+1}$ to derive the second inequality above. Namely, we have from (3.8) and (3.9) that

$$
\begin{aligned}
& \frac{1}{2} m^{2}(2 \delta)^{-2 m-n} \int_{|x| \leq 2 \delta}|u|^{2} d x+m^{2}\left(R_{0}^{2} R_{j}\right)^{-2 m-n} \int_{|x| \leq R_{0}^{2} R}|u|^{2} d x \\
& \leq \tilde{C}^{\prime \prime} \delta^{-2 m-n} \int_{|x| \leq \delta}|u|^{2} d x \\
& \quad+m^{2}\left(R_{0}^{2} R_{j}\right)^{-2 m-n} \exp \left(-c R^{-2 \epsilon / l}\right) \int_{|x| \leq R}|u|^{2} d x .
\end{aligned}
$$

If there exists $s \in \mathbb{N}$ such that

$$
R_{j+1}<R_{0}^{2 s} \leq R_{j} \quad \text { for some } \quad j \geq j_{0},
$$

then replacing $R$ by $R_{0}^{2 s}$ in (3.10) leads to

$$
\begin{aligned}
& \frac{1}{2} m^{2}(2 \delta)^{-2 m-n} \int_{|x| \leq 2 \delta}|u|^{2} d x+m^{2}\left(R_{0}^{2} R_{j}\right)^{-2 m-n} \int_{|x| \leq R_{0}^{2 s+2}}|u|^{2} d x \\
& \leq \tilde{C}^{\prime \prime} \delta^{-2 m-n} \int_{|x| \leq \delta}|u|^{2} d x \\
& \quad+m^{2}\left(R_{0}^{2} R_{j}\right)^{-2 m-n} \exp \left(-c R_{0}^{-4 s \epsilon / l}\right) \int_{|x| \leq R_{0}^{2 s}}|u|^{2} d x .
\end{aligned}
$$


Here $s$ and $R_{0}$ are yet to be determined. The trick now is to find suitable $s$ and $R_{0}$ satisfying (3.11) such that the inequality

$$
\exp \left(-c R_{0}^{-4 s \epsilon / l}\right) \int_{|x| \leq R_{0}^{2 s}}|u|^{2} d x \leq \frac{1}{2} \int_{|x| \leq R_{0}^{2 s+2}}|u|^{2} d x
$$

holds with such choices of $s$ and $R_{0}$.

It is time to use the three-sphere inequality (1.2). To this end, we choose $r_{1}=$ $R_{0}^{2 k+2}, r_{2}=R_{0}^{2 k}$ and $r_{3}=R_{0}^{2 k-2}$ for $k \geq 1$. Note that $r_{1} / r_{3}<r_{2} / r_{3} \leq R_{0}^{2} \leq \tilde{R}_{0}$. Thus (1.2) implies

$$
\begin{aligned}
& \int_{|x|<R_{0}^{2 k}}|u|^{2} d x / \int_{|x|<R_{0}^{2 k+2}}|u|^{2} d x \\
& \leq C^{1 / \tau}\left(\int_{|x|<R_{0}^{2 k-2}}|u|^{2} d x / \int_{|x|<R_{0}^{2 k}}|u|^{2} d x\right)^{a},
\end{aligned}
$$

where

$$
C=\max \left\{C_{0} R_{0}^{-2 n}, \exp \left(\beta_{0}\left(-1-4 \log R_{0}\right)\right)\right\}
$$

and

$$
\begin{aligned}
a=\frac{1-\tau}{\tau}=\frac{A}{B} & =\frac{\left(\log \left(r_{1} / r_{3}\right)-1\right)^{2}-\left(\log \left(r_{2} / r_{3}\right)\right)^{2}}{-1-2 \log \left(r_{2} / r_{3}\right)} \\
& =\frac{\left(4 \log R_{0}-1\right)^{2}-\left(2 \log R_{0}\right)^{2}}{-1-4 \log R_{0}} .
\end{aligned}
$$

It is not hard to see that

$$
\left\{\begin{array}{l}
1<C \leq C_{0} R_{0}^{-\beta_{1}}, \\
2<a \leq-4 \log R_{0},
\end{array}\right.
$$

where $\beta_{1}=\max \left\{2 n, 4 \beta_{0}\right\}$ and if $R_{0}$ is sufficiently small, e.g., $R_{0} \leq e^{-4}$. Combining (3.15) and using (3.14) recursively, we have

$$
\begin{aligned}
& \int_{|x| \leq R_{0}^{2 s}}|u|^{2} d x / \int_{|x| \leq R_{0}^{2 s+2}}|u|^{2} d x \\
& \leq C^{1 / \tau}\left(\int_{|x|<R_{0}^{2 s-2}}|u|^{2} d x / \int_{|x|<R_{0}^{2 s}}|u|^{2} d x\right)^{a} \\
& \leq C^{\frac{a^{s-1}-1}{\tau(a-1)}}\left(\int_{|x|<R_{0}^{2}}|u|^{2} d x / \int_{|x|<R_{0}^{4}}|u|^{2} d x\right)^{a^{s-1}}
\end{aligned}
$$


for all $s \geq 1$. Now from the definition of $a$, we have $\tau=1 /(a+1)$ and thus

$$
\frac{a^{s-1}-1}{\tau(a-1)}=\frac{a+1}{a-1}\left(a^{s-1}-1\right) \leq 3 a^{s-1} .
$$

Then it follows from (3.16) that

$$
\begin{aligned}
& \int_{|x| \leq R_{0}^{2 s}}|u|^{2} d x / \int_{|x| \leq R_{0}^{2 s+2}}|u|^{2} d x \\
& \leq C^{3\left(-4 \log R_{0}\right)^{s-1}}\left(\int_{|x|<R_{0}^{2}}|u|^{2} d x / \int_{|x|<R_{0}^{4}}|u|^{2} d x\right)^{a^{s-1}} \\
& \leq\left(C_{0}^{3}\left(R_{0}\right)^{-3 \beta_{1}}\right)^{\left(-4 \log R_{0}\right)^{s-1}}\left(\int_{|x|<R_{0}^{2}}|u|^{2} d x / \int_{|x|<R_{0}^{4}}|u|^{2} d x\right)^{a^{s-1}} .
\end{aligned}
$$

Thus, by (3.17), we can get that

$$
\begin{aligned}
& \exp \left(-c R_{0}^{-4 s \epsilon / l}\right) \int_{|x| \leq R_{0}^{2 s}}|u|^{2} d x \\
& \leq \exp \left(-c R_{0}^{-4 s \epsilon / l}\right)\left(C_{0}^{3}\left(R_{0}\right)^{-3 \beta_{1}}\right)^{\left(-4 \log R_{0}\right)^{s-1}} \\
& \left(\int_{|x|<R_{0}^{2}}|u|^{2} d x / \int_{|x|<R_{0}^{4}}|u|^{2} d x\right)^{a^{s-1}} \int_{|x| \leq R_{0}^{2 s+2}}|u|^{2} d x .
\end{aligned}
$$

Let $\mu=-\log R_{0}$. Then if $R_{0}\left(\leq \min \left\{e^{-4}, \sqrt{\tilde{R}_{0}}\right\}\right)$ is sufficiently small, i.e., $\mu$ is sufficiently large, we can see that

$$
4 t \epsilon \mu / l>(t-1) \log (4 \mu)+\log \left(\log C_{0}^{3}+3 \beta_{1} \mu\right)-\log (c / 4),
$$

for all $t \in \mathbb{N}$. In other words, for small $R_{0}$ we have that

$$
\left(C_{0}^{3} R_{0}^{-3 \beta_{1}}\right)^{\left(-4 \log R_{0}\right)^{t-1}}<\exp \left(c R_{0}^{-4 t \epsilon / l} / 4\right)<(1 / 2) \exp \left(c R_{0}^{-4 t \epsilon / l} / 2\right),
$$

for all $t \in \mathbb{N}$. We now fix such an $R_{0}$ so that (3.19) holds and

$$
-\frac{4 \varepsilon}{l} \log R_{0}-2 \log a>0 .
$$

It is a key step in our proof that we can find a universal constant $R_{0}$. After fixing $R_{0}$, we then define a number $t_{0}$, depending on $R_{0}$ and $u$, as

$$
\begin{aligned}
t_{0}= & \left(\log 2-\log (a c)+\log \log \left(\int_{|x|<R_{0}^{2}}|u|^{2} d x / \int_{|x|<R_{0}^{4}}|u|^{2} d x\right)\right) \\
& \times\left(-\frac{4 \varepsilon}{l} \log R_{0}-\log a\right)^{-1} .
\end{aligned}
$$


With this choice of $t_{0}$, we can see that

$$
\left(\int_{|x|<R_{0}^{2}}|u|^{2} d x / \int_{|x|<R_{0}^{4}}|u|^{2} d x\right)^{a^{t-1}} \leq \exp \left(c R_{0}^{-4 t \epsilon / l} / 2\right)
$$

for all $t \geq t_{0}$. Let $s_{1}$ be the smallest positive integer such that $s_{1} \geq t_{0}$. If

$$
R_{0}^{2 s_{1}} \leq R_{j_{0}}=\left(\gamma\left(j_{0}+1 / 2\right)\right)^{-l / 2 \epsilon}
$$

then we can find $j_{1} \in \mathbb{N}$ with $j_{1} \geq j_{0}$ such that (3.11) holds, i.e.,

$$
R_{j_{1}+1}<R_{0}^{2 s_{1}} \leq R_{j_{1}}
$$

On the other hand, if

$$
R_{0}^{2 s_{1}}>R_{j_{0}}
$$

then we pick the smallest positive integer $s_{2}>s_{1}$ such that $R_{0}^{2 s_{2}} \leq R_{j_{0}}$ and thus we can also find $j_{1} \in \mathbb{N}$ with $j_{1} \geq j_{0}$ for which (3.11) holds. We now define

$$
s=\left\{\begin{array}{llll}
s_{1} & \text { if } & (3.21) & \text { holds } \\
s_{2} & \text { if } & (3.22) & \text { holds }
\end{array}\right.
$$

It is important to note that with such an $s,(3.11)$ is satisfied for some $j_{1}$ and (3.19), (3.20) hold. Therefore, we set $m_{1}=n+2\left(j_{1}+1 / 2\right)$ and $m=\left(m_{1}-n\right) / 2$. Combining (3.18), (3.19) and (3.20) yields

$$
\begin{aligned}
& \exp \left(-c R_{0}^{-4 s \epsilon / l}\right) \int_{|x| \leq R_{0}^{2 s}}|u|^{2} d x \\
& \leq \exp \left(-c R_{0}^{-4 s \epsilon / l}\right)\left(C_{0}^{3}\left(R_{0}\right)^{-3 \beta_{1}}\right)^{\left(-3 \log R_{0}\right)^{s-1}} \\
& \left(\int_{|x|<R_{0}^{2}}|u|^{2} d x / \int_{|x|<R_{0}^{4}}|u|^{2} d x\right)^{a^{(s-1)}} \int_{|x| \leq R_{0}^{2 s+2}}|u|^{2} d x \\
& \leq \frac{1}{2} \int_{|x| \leq R_{0}^{2 s+2}}|u|^{2} d x
\end{aligned}
$$


which is (3.13). Using (3.13) in (3.12), we have

$$
\begin{aligned}
& \frac{1}{2} m^{2}(2 \delta)^{-2 m-n} \int_{|x| \leq 2 \delta}|u|^{2} d x+\frac{1}{2} m^{2}\left(R_{0}^{2} R_{j_{1}}\right)^{-2 m-n} \int_{|x| \leq R_{0}^{2 s+2}}|u|^{2} d x \\
& \leq \tilde{C}^{\prime \prime} \delta^{-2 m-n} \int_{|x| \leq \delta}|u|^{2} d x .
\end{aligned}
$$

From (3.23), we get

$$
\frac{\left(m_{1}-n\right)^{2}}{8 \tilde{C}^{\prime \prime}}\left(R_{0}^{2} R_{j_{1}}\right)^{-m_{1}} \int_{|x| \leq R_{0}^{2 s+2}}|u|^{2} d x \leq \delta^{-m_{1}} \int_{|x| \leq \delta}|u|^{2} d x
$$

and

$$
\frac{1}{2} m^{2}(2 \delta)^{-2 m-n} \int_{|x| \leq 2 \delta}|u|^{2} d x \leq \tilde{C}^{\prime \prime} \delta^{-2 m-n} \int_{|x| \leq \delta}|u|^{2} d x
$$

which implies

$$
\int_{|x| \leq 2 \delta}|u|^{2} d x \leq \frac{8 \tilde{C}^{\prime \prime}}{\left(m_{1}-n\right)^{2}} 2^{m_{1}} \int_{|x| \leq \delta}|u|^{2} d x .
$$

The estimates (3.24) and (3.25) are valid for all $\delta \leq R_{0}^{2 s+2} / 4$. Therefore, (1.3) holds with $R_{2}=R_{0}, R_{3}=R_{0}^{2 s+2} / 4$ and $C_{3}=\frac{\left(m_{1}-n\right)^{2}}{8 \tilde{C}^{\prime \prime}}\left(R_{0}^{2} R_{j_{1}}\right)^{-m_{1}} \int_{|x| \leq R_{0}^{2 s+2}}|u|^{2} d x$. Moreover (1.4) holds with $R_{4}=R_{0}^{2 s+2} / 8$ and $C_{4}=\frac{8 \tilde{C}^{\prime \prime}}{\left(m_{1}-n\right)^{2}} 2^{m_{1}}$ and the proof is now complete. Here we have proved (1.3) and (1.4) together. In fact, (1.3) can be seen as a corollary of (1.4) (see, for example, [6, page 135]).

\section{References}

[1] G. Alessandrini, E. Beretta, E. Rosset and S. Vessella, Optimal stability for elliptic boundary value problems with unknow boundaries, Ann. Sc. Norm. Super. Pisa Cl. Sci. 29 (2000), 755-786.

[2] F. Colombini and C. GRAmmatico, Some remarks on strong unique continuation for the Laplacian and its powers, Comm. Partial Differential Equations 24 (1999), 1079-1094.

[3] H.DONNELLY and C. FEFFERMAN, Nodal sets of eigenfunctions on Riemannian manifolds, Invent. Math. 93 (1988), 161-183.

[4] N. GAROFALO and F. H. LIN, Monotonicity properties of variational integrals, $A_{p}$ weights and unique continuation, Indiana Univ. Math. J. 35 (1986), 245-267.

[5] N. GAROFAlO and F. H. LIN, Unique continuation for elliptic operators: a geometricvariational approach, Comm. Pure Appl. Math. 40 (1987), 347-366.

[6] M. Giaquinta, "Multiple Integrals in the Calculus of Variations and Nonlinear Elliptic Systems", Annals of Mathematics Studies, Vol. 105, Princeton University Press, NJ, 1983. 
[7] R. Hardt and L. Simon, Nodal sets for solutions of elliptic equations, J. Differential Geom. 30 (1989), 505-522.

[8] L. HöRmANDER "The Analysis of Linear Partial Differential Operators", Vol. 3, SpringerVerlag, Berlin/New York, 1985.

[9] C. L. LIN, Strong unique continuation for $m$-th powers of a Laplaceian operator with singular coefficients, Proc. Amer. Math. Soc. 135 (2007), 569-578.

[10] C. L. LIN, G. NAKAMURA and J.-N. WANG, Quantitative uniqueness for second-order elliptic operators with strongly singular coefficients, Rev. Mat. Iberoam. 27 (2011), 475491.

[11] F. H. Lin, Nodal sets of solutions of elliptic and parabolic equations, Comm. Pure Appl. Math. 44 (1991), 287-308.

[12] M. Protter, Unique continuation for elliptic equations, Trans. Amer. Math. Soc. 95 (1960), 81-91.

\author{
Department of Mathematics \\ NCTS, National Cheng Kung University \\ Tainan 701, Taiwan \\ cllin2@mail.ncku.edu.tw \\ Department of Mathematics \\ Taida Institute for Mathematical Sciences \\ National Taiwan University \\ Taipei 106, Taiwan \\ nagayasu@math.ntu.edu.tw \\ Department of Mathematics \\ Taida Institute of Mathematical Sciences \\ NCTS (Taipei), National Taiwan University \\ Taipei 106, Taiwan \\ jnwang@math.ntu.edu.tw
}

